\title{
INVARIANT FACTORS AND TWO CRITERIA FOR PRO JECTIVITY OF MODULES
}

\author{
BY \\ MAURICE AUSLANDER AND DAVID A. BUCHSBAUM( $\left.{ }^{1}\right)$
}

Let $R$ be a commutative ring, and $E$ an $R$-module. For each $p \geqq 1$, we define the ideal $\alpha_{p}(E)$ to be the annihilator of the $p$ th exterior product of $E$ (denoted by $\Lambda^{p} E$ ), and $\alpha_{p}(E)$ is called the pth invariant factor of $E$. If $E$ is a finitely generated $R$-module, then $\Lambda^{p} E=0$ (or $\alpha_{p}(E)=R$ ) for all but a finite number of $p$, and the largest integer $p$ for which $\Lambda^{p} E \neq 0$ is called the exterior rank of $E$ (denoted by ext rank $E$ ).

The fact that the invariant factors of a module give some insight into the structure of the module $\left({ }^{2}\right)$ is shown in $\S 1$, where we prove

TheOREM A. Let $R$ be a local domain, and $E$ a finitely generated $R$-module. If $\alpha_{p}(E)$ is a principal ideal for all $p$, then $E=\oplus_{p} R / \alpha_{p}(E)$ (direct sum).

By investigating some of the properties of $\alpha_{s}(E)$, where $s=\operatorname{ext}$ rank $E$, we obtain, in $\S 3$, proofs of the following two theorems:

TheOREM B. Let $R$ be a noetherian ring $\left({ }^{3}\right)$, and $E$ a finitely generated $R$ module. If the dimension of the $R / \mathfrak{m}$ vector space $E / \mathfrak{m} E$ is the same for each maximal ideal $\mathfrak{m}$ in $R$, and if $\mathfrak{r}$ denotes the radical of $R$, then $E / \mathfrak{r} E$ is $R / \mathfrak{r}$ projective. If $R / \mathfrak{r}$ is a domain, then this sufficient condition is also necessary.

Theorem C. If $R$ is a local domain, $E$ a finitely generated $R$-module, and $\mathfrak{p}$ a prime ideal in $R$ such that $E / \mathfrak{p} E$ is $R / \mathfrak{p}$-free and $E_{\mathfrak{p}}$ is $R_{\mathfrak{p}}$-free, then $E$ is $R$ free.

In $\$ 3$ we consider another ideal, $\beta(E)$, associated with an $R$-module $E$, and indicate its connection with the invariant factors $\alpha_{p}(E)$. We also indicate how $\beta(E)$ may be used to give an alternate proof to Theorem $C$.

1. Proof of Theorem A. In this section, we are assuming that $R$ is a local ring, and that $E$ is a finitely generated $R$-module. We recall that if $\bar{x}_{1}, \cdots, \bar{x}_{n}$ is a basis for $E / \mathfrak{m} E$ over $R / \mathfrak{m}$ (where $\mathfrak{m}$ is the maximal ideal of $R$ ), then $x_{1}, \cdots, x_{n}$ is a minimal generating set of $E$ over $R$ if $x_{i}$ in $E$ represents the coset of $\bar{x}_{i} \in E / \mathrm{m} E$. Moreover, since $\Lambda^{p} E / \mathrm{m} E$ is the same whether con-

Received by the editors October 11, 1961.

(1) This work was one with the partial support of NSF Grant G-5864.

(2) S. Kertzner is systematically studying the invariant factors of modules over regular local rings to see the extent to which they determine the structure of such modules.

(3) Throughout this note our rings will be commutative with identity, and the radical will be the intersection of the maximal ideals. Modules will be unitary; if $E$ is an $R$-module and $p$ a prime ideal, we denote by $\Lambda^{p} E, R_{\mathfrak{p}}, E_{\mathfrak{p}}$ respectively the $p$ th exterior product, the ring of quo. tients of $R$ with respect to $\mathfrak{p}$, and the modules of quotients of $E$ with respect to $\mathfrak{p}$. 
sidered as an $R$-module or an $R / \mathfrak{m}$-module, we know that $\Lambda^{p} E / \mathfrak{m} E \neq 0$ for $0 \leqq p \leqq n$, and $\Lambda^{p} E / \mathfrak{m} E=0$ for $p>n$. From the fact that $\Lambda^{p} E \rightarrow \Lambda^{p} E / \mathfrak{m} E$ is an epimorphism for each $p$, we conclude that $\Lambda^{p} E \neq 0$ for $0 \leqq p \leqq n$; clearly $\Lambda^{p} E=0$ for $p>n$. Thus for local rings, ext rank $E=\operatorname{dim}_{R / \mathfrak{m}} E / \mathfrak{m} E$.

The foregoing remarks make the computation of $\alpha_{n}(E)$ rather easy, if $n=\operatorname{ext} \operatorname{rank} E$. For let

$$
0 \rightarrow K \rightarrow F \rightarrow E \rightarrow 0
$$

be an exact sequence of $R$-modules with $F$ free of rank $n=\operatorname{ext}$ rank $E$. Let $y_{1}, \cdots, y_{n}$ be a basis of $F$, and $z_{1}, \cdots, z_{m}$ a set of generators for $K$. If $z_{i}=\sum a_{i j} y_{j}$, then $\alpha_{n}(E)$ is the ideal generated by the $a_{i j}$.

Using these preliminary observations, we can now prove the following lemma.

LEMma 1.1. Let $R$ be a local domain, and $E$ a finitely generated $R$-module of exterior rank $n$. If $\alpha_{n}(E)$ is a principal ideal, then $E \approx R / \alpha_{n}(E) \oplus E^{\prime}$.

Proof. Let $\alpha_{n}(E)=(\epsilon)$ and let the $a_{i j}$ be the coefficients arising from the exact sequence (1). Since $\alpha_{n}(E)$ is generated by the $a_{i j}$, and since any generating set for a module over a local ring contains a minimal generating set, we may assume that $\left(a_{11}\right)=(\epsilon)$ and, for convenience, we shall assume that $a_{11}=\epsilon$.

Setting $a_{i j}=a_{1,}^{\prime} \epsilon$, with $a_{11}^{\prime}=1$, let $y_{1}^{\prime}=y_{1}+\sum_{j=2}^{n} a_{1 j}^{\prime} y_{j}$. Then $y_{1}^{\prime}, y_{2}, \cdots, y_{n}$ is a basis for $F$, and $z_{1}=\epsilon y_{1}^{\prime}$. Let $F^{\prime}$ be the submodule of $F$ generated by $y_{2}, \cdots, y_{n}$, and $K^{\prime}=K \cap F^{\prime}$. It is easy to see that $K=\left(z_{1}\right) \oplus K^{\prime}$, so that $E=F / K=R /(\epsilon) \oplus F^{\prime} / K^{\prime}$.

The proof of Theorem A proceeds by induction on ext rank $E$, the case of ext rank $E=1$ being trivial. If $n=\operatorname{ext} \operatorname{rank} E$, and if we assume that all the invariant factors of $E$ are principal, then Lemma 1.1 tells us that $E=R / \alpha_{n}(E)$ $\oplus E^{\prime}$, and all we need show is that ext rank $E^{\prime}=n-1$ and $\alpha_{p}\left(E^{\prime}\right)=\alpha_{p}(E)$ for $p<n$. Clearly ext rank $E^{\prime}=n-1$. To see that $\alpha_{p}(E)=\alpha_{p}\left(E^{\prime}\right)$ for $p<n$, we use the fact that $\Lambda^{p} E \approx R / \alpha_{n}(E) \otimes \Lambda^{p-1} E^{\prime} \oplus \Lambda^{p} E^{\prime}$, so that $\alpha_{p}(E)=\alpha_{p}\left(E^{\prime}\right)$ $\operatorname{Cann}\left(R / \alpha_{n}(E) \otimes \Lambda^{p-1} E^{\prime}\right)$. Now $\alpha_{n}\left(E^{\prime}\right)=R$, and $\alpha_{n}(E) \subset\left(\alpha_{n}(E), \alpha_{n-1}\left(E^{\prime}\right)\right)$ $\operatorname{Cann}\left(R / \alpha_{n}(E) \otimes \bigwedge^{n-1} E^{\prime}\right)=R \cap \operatorname{ann}\left(R / \alpha_{n}(E) \otimes \bigwedge^{n-1} E^{\prime}\right)=\alpha_{n}(E)$. Therefore we must have $\alpha_{n-1}\left(E^{\prime}\right) \subset \alpha_{n}(E)$. But then using $\alpha_{n-1}(E)=\alpha_{n-1}\left(E^{\prime}\right)$ $\operatorname{nann}\left(R / \alpha_{n}(E) \otimes \bigwedge^{n-2} E^{\prime}\right)$ we see that $\alpha_{n-1}\left(E^{\prime}\right)=\alpha_{n-1}(E)$. Proceeding in this way, and using the relation of $\alpha_{p}\left(E^{\prime}\right) \subset \alpha_{n-1} E^{\prime} \subset \alpha_{n}(E)$ for all $p$, we obtain $\alpha_{p}\left(E^{\prime}\right)=\alpha_{p}(E)$ for $p<n$. Hence we have completed the inductive step and Theorem $\mathrm{A}$ is proved.

2. The ideal $\alpha(E)$. Let $R$ be a commutative ring and $E$ a finitely generated $R$-module. For each maximal ideal $\mathfrak{m}$ in $R$, the dimension of the $R / \mathfrak{m}$ vector space $E / \mathfrak{m} E$ (notation: $\operatorname{dim}(E / \mathfrak{m} E)$ ) is less than or equal to the least number of elements necessary to generate $E$ over $R$. Thus the set of integers $\operatorname{dim}(E / \mathrm{m} E)$ is bounded where $\mathfrak{m}$ runs through all maximal ideals $\mathfrak{m}$ in $E$. We call the least 
upper bound of this set the residual rank of $E$ (notation: res rank $E$ ).

An elementary but useful remark on the residual rank of a module is the following:

Lemma 2.1. A maximal ideal $\mathfrak{m}$ in $R$ contains the annihilator of $E$ if and only if $E / \mathfrak{m} E \neq 0$. Therefore $E=0$ if and only if $E / \mathfrak{m} E=0$ for all maximal ideals $\mathfrak{m}$ in $R$. Thus $E$ has residual rank zero if and only if $E=0$.

Proof. Let $\mathfrak{m}$ be a maximal ideal in $R$. It follows from the usual properties of rings of quotients, that $E / \mathfrak{m} E=E_{\mathfrak{m}} / \mathfrak{m} R_{\mathfrak{m}}\left(E_{\mathfrak{m}}\right)$. Now $E$ is a finitely generated $R$-module and therefore $E_{\mathfrak{m}}$ is a finitely generated $R_{\mathfrak{m}}$-module. Since $\mathfrak{m} R_{\mathfrak{m}}$ is the radical of $R_{\mathfrak{m}}$, it follows from Nakayama's lemma that ( $\left.\mathfrak{m} R_{\mathfrak{m}}\right) E_{\mathfrak{m}}$ $=E_{\mathfrak{m}}$ if and only if $E_{\mathfrak{m}}=0$. Appealing once more to the fact that $E$ is a finitely generated $R$-module, we have that $E_{\mathfrak{m}}=0$ if and only if $\mathfrak{m}$ does not contain the annihilator of $E$. The lemma is now easily established.

If $s$ is the exterior rank of $E$, then we denote the annihilator of $\Lambda^{*} E$ by $\alpha(E)$, (i.e. $\alpha(E)=\alpha_{s}(E)$ ).

Proposition 2.2. For a finitely generated $R$-module $E$ we have,

(a) The ext rank $E=\operatorname{res} \operatorname{rank} E$,

(b) for a maximal ideal $\mathfrak{m}$, the $\operatorname{dim}(E / \mathfrak{m} E)=\operatorname{ext} \operatorname{rank} E$ if and only if $\mathfrak{m}$ contains $\alpha(E)$.

Proof. If we denote the exterior algebra of $E$ over $R$ by $\varepsilon_{R}(E)$, it is well known that $R / \mathfrak{a} \otimes_{R} \varepsilon_{R}(E)=\varepsilon_{R / \mathfrak{a}}(E / \mathfrak{a} E)$ for any ideal $\mathfrak{a}$ in $R$, (see [3, V, Theorem 52]). Now for a maximal ideal $m$ in $R$, it is well known that $\operatorname{dim}(E / \mathfrak{m} E)=$ ext $\operatorname{rank} E / \mathfrak{m} E$. Since $R / \mathfrak{m} \otimes \mathcal{E}_{R}(E)=\mathcal{E}_{R / \mathfrak{m}}(E / \mathfrak{m} E)$, we have that the ext rank $E \geqq$ res rank $E$.

Suppose $s=$ ext rank $E$. Then $\Lambda^{8} E \neq 0$ and is a finitely generated $R$ module. Therefore applying Lemma 2.1 , we know that for some maximal ideal $\mathfrak{m}$ in $R$, we have $R / \mathfrak{m} \otimes \Lambda^{*} E \neq 0$. But $R / \mathfrak{m} \otimes \Lambda^{s} E=\Lambda^{*} E / \mathfrak{m} E$, which shows that the $\operatorname{dim}(E / \mathfrak{m} E)=s$. Therefore the ext rank $E=$ res rank $E$.

Part (b) of the proposition also follows immediately from Lemma 2.1 in a similar manner.

In the next few propositions we establish some of the basic properties of $\alpha(E)$ in the case that $R$ is a local ring, i.e. $R$ has only one maximal ideal.

The first part of the following proposition has already been discussed in $\S 1$. However, we include the entire statement for the sake of completeness.

Proposition 2.3. Let $R$ be a local ring with maximal ideal $\mathfrak{m}$ and $E$ a finitely generated $R$-module. Then $\Lambda^{n} E \approx R / \alpha(E)$ and $E$ is free if and only if $\alpha(E)=0$.

Proof. Let $n=\operatorname{dim}(E / \mathfrak{m} E)$ where $\mathfrak{m}$ is the maximal ideal in $R$. Let $x_{1}, \cdots, x_{n}$ be elements in $E$ whose images in $E / \mathfrak{m} E$ are a basis for the $R / \mathfrak{m}$ vector space $E / \mathfrak{m} E$. Then by Nakayama's lemma we have that $x_{1}, \cdots, x_{n}$ 
generate $E$. Let $F$ be a free $R$-module with basis $y_{1}, \cdots, y_{n}$ and define the epimorphism $f: F \rightarrow E$ by $f\left(y_{i}\right)=x_{i}$. Then $f$ induces an epimorphism $\varepsilon(F)$ $\rightarrow \mathcal{E}(E)$. Therefore we have that $\Lambda^{n} E=R / \alpha(E)$.

If $E$ is free, then $x_{1}, \cdots, x_{n}$ are a basis for $E$ over $R$ (see [2, VIII, Theorem 6.2]) and therefore $\Lambda^{n} E \approx R$ which means that $\alpha(E)=0$. On the other hand if $E$ is not free then some multiple of one of the $x_{i}$ can be written as a linear combination of the others. Let us say that $r x_{1}=\sum_{j=2}^{n} r_{j} x_{j}$ with $r \neq 0$. Since the $x_{j}$ are linearly independent $\bmod \mathfrak{m}$, we have that $r$ is in $\mathfrak{m}$. Now $x_{1} \wedge \cdots \wedge x_{n}$ generates $\wedge^{n} E$ and

$r\left(x_{1} \wedge \cdots \wedge x_{n}\right)=$

$$
\left(\sum_{j=2}^{n} r_{j} x_{j}\right) \wedge x_{2} \wedge \cdots \wedge x_{n}=\sum_{j=2}^{n} r_{j}\left(x_{j} \wedge x_{2} \wedge \cdots \wedge x_{n}\right)=0
$$

since each $x_{j} \wedge x_{2} \wedge \cdots \wedge x_{n}=0$ for $j>1$. Therefore we have $\alpha(E) \neq 0$.

Corollary 2.4. Let $R$ be a local ring with maximal ideal $\mathfrak{m}$ and $E$ a finitely generated $R$-module. Then an ideal $\mathfrak{a}$ in $R$ contains $\alpha(E)$ if and only if $E / \mathfrak{a} E$ is $R / \mathfrak{a}$-free.

Proof. Let $\mathfrak{a}$ be an ideal in $R$. Then $\varepsilon_{R / \mathfrak{a}}(E / \mathfrak{a} E)=R / \mathfrak{a} \otimes \mathcal{E}_{R}(E)$. Since the exterior rank of $E$ and $E / \mathfrak{a} E$ are the same, let us say $n$, we have that $\Lambda^{n} E / \mathfrak{a} E \approx R / \mathfrak{a} \otimes \bigwedge^{n} E=R / \mathfrak{a} \otimes R / \alpha(E)=R /(\mathfrak{a}, \alpha(E))$. Now by Proposition 2.3 we know that $E / \mathfrak{a} E$ is $R / \mathfrak{a}$-free if and only if $R /(\mathfrak{a}, \alpha(E)) \approx R / \mathfrak{a}$. It therefore follows that $E / \mathfrak{a} E$ is $R / \mathfrak{a}$-free if and only if $\mathfrak{a} \supset \alpha(E)$.

3. Proof of Theorems B and C. Before proceeding to the proof of Theorem $\mathrm{B}$ we establish the following proposition from which the theorem will follow easily.

Proposition 3.1. Let $E$ be a finitely generated $R$-module ( $R$ not necessarily local). Then for each ideal $\mathfrak{b} \supset \alpha(E)$ we have that $E / \mathfrak{b} E$ is $R / \mathfrak{b}$-flat.

Proof. Suppose the ideal $\mathfrak{b}$ in $R$ contains $\alpha(E)$. To show that $E / \mathfrak{b} E$ is $R / \mathfrak{b}$-flat it suffices to show that $\operatorname{Tor}_{1}^{R / \mathfrak{b}}(E / \mathfrak{b} E, C)=0$ for all $R / \mathfrak{b}$-modules $C$. Now if we show that for each maximal ideal $\mathfrak{m} \supset \mathfrak{b}$ we have that $(R / \mathfrak{b})_{\mathfrak{m}} \otimes_{R} \operatorname{Tor}_{1}^{R / \mathfrak{b}}(E / \mathfrak{b} E, C)=\operatorname{Tor}_{1}^{(R / \mathfrak{b})} \mathfrak{m}\left((E / \mathfrak{b} E)_{\mathfrak{m}}, C_{\mathfrak{m}}\right)=0$, then it will follow that $\operatorname{Tor}_{1}^{R / \mathfrak{b}}(E / \mathfrak{b} E, C)=0$ and we will be done (see [2, VII, Exercises 10 and 11]). This will certainly be the case if we show that for each maximal ideal $\mathfrak{m} \supset \mathfrak{b}$ we have that $(E / \mathfrak{b} E)_{\mathfrak{m}}$ is $(R / \mathfrak{b})_{\mathfrak{m}}$-free, which is what we now proceed to do.

Suppose the ext rank $E=n$. Then if $\mathfrak{m} \supset \mathfrak{b} \supset \alpha(E)$ we have by Lemma 2.1 that $\operatorname{dim}(E / \mathfrak{m} E)=n$. Now $R_{\mathfrak{m}} \otimes \mathcal{E}_{R}(E)=\mathcal{E}_{R_{\mathfrak{m}}}\left(E_{\mathfrak{m}}\right)$ (see [3; V, Theorem 52]). Also the fact that $E$ is a finitely generated $R$-module tells us that $\varepsilon_{R}(E)$ is a finitely generated $R$-module. Therefore we have that $\alpha(E)_{\mathfrak{m}}$ is the annihilator of $R_{\mathfrak{m}} \otimes \Lambda^{n} E=\Lambda^{n} E_{\mathfrak{m}}$ since $\alpha(E)$ is the annihilator of $\Lambda^{n} E$ (see [1, Lemma 
2.6]). Because $\mathfrak{b} \supset \alpha(E)$ we have that $\mathfrak{b}_{\mathfrak{m}} \supset \alpha(E)_{\mathfrak{m}}$ and thus by Corollary 2.4 we have that $(E / \mathfrak{b} E)_{\mathfrak{m}}=E_{\mathfrak{m}} /\left(\mathfrak{b}_{\mathfrak{m}}\right) E_{\mathfrak{m}}$ is $(R / \mathfrak{b})_{\mathfrak{m}}$-free.

Corollary 3.2. Let $R$ be a ring and let $\mathfrak{r}$ be its radical. If $E$ is a finitely generated $R$-module such that $\operatorname{dim}(E / \mathfrak{m} E)$ is the same for all maximal ideals $\mathfrak{m}$ in $R$, then $E / \mathfrak{r} E$ is $R / \mathfrak{r}$-flat.

Proof. Since the dimension of $E / \mathfrak{m} E$ is independent of $\mathfrak{m}$ we have by Proposition 2.2, that each maximal ideal $\mathfrak{m} \supset \alpha(E)$. Since the intersection of the maximal ideals is $\mathfrak{r}$, we have that $\alpha(E) \subset \mathfrak{r}$. The corollary now follows from Proposition 2.1.

Now if we add to Corollary 3.2 the hypothesis that $R$ is noetherian we have the hypothesis of the first part of Theorem B. Since over a noetherian ring a finitely generated flat-module is projective (see [2, VI, Example 3]), we have established the first part of Theorem $\mathrm{B}$. Now suppose $R=R / \mathfrak{r}$ is a noetherian domain, and $E$ is a finitely generated $R$-module such that $\bar{E}=E / \mathfrak{r} E$ is $\bar{R}$-projective. Then for every maximal ideal $\mathfrak{m}$ in $R$, we have that

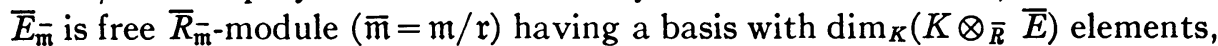
where $K$ is the quotient field of $\bar{R}$. Since $E / \mathfrak{m} E \approx \bar{E}_{\overline{\mathfrak{m}}} /\left(\overline{\mathrm{m}} \bar{R}_{\overline{\mathrm{m}}}\right) \bar{E}_{\overline{\mathrm{m}}}$, we have that $\operatorname{dim} E / \mathfrak{m} E=$ rank of the free $\bar{R}_{\overline{\mathfrak{m}}}$-module $\bar{E}_{\overline{\mathfrak{m}}}$ which is independent of the maximal ideal $m$ that was chosen. This completes the proof of Theorem B.

We now come to the proof of Theorem C. By Proposition 2.3, it suffices to show that $\alpha(E)=0$. Now since we are given that $E / \mathfrak{p} E$ is $R / \mathfrak{p}$-free, we see from Corollary 2.4 that $\mathfrak{p}$ contains $\alpha(E)$. Furthermore, from the facts that $R_{\mathfrak{p}} \otimes \Lambda^{p} E \approx \Lambda^{p} E_{\mathfrak{p}}$ and that for any finitely generated $R$-module $M$ the annihilator of $M_{\mathfrak{p}}$ is the extension to $R_{\mathfrak{p}}$ of the annihilator of $M$, we see that if $\alpha(E)=\alpha_{n}(E)$, then $\alpha_{n}\left(E_{\mathfrak{p}}\right)=\alpha(E) \cdot R_{\mathfrak{p}}$. Thus, since $\alpha(E)$ is contained in $\mathfrak{p}$, $\alpha(E) R_{\mathfrak{p}} \neq R_{\mathfrak{p}}$, so that $\alpha_{n}\left(E_{\mathfrak{p}}\right) \neq R_{\mathfrak{p}}$. However, the number of generators of $E_{\mathfrak{p}}$ over $R_{\mathfrak{p}}$ does not exceed that of $E$ over $R$, so we may conclude that $\alpha_{n}\left(E_{\mathfrak{p}}\right)$ $=\alpha\left(E_{\mathfrak{p}}\right)$.

But by the hypotheses of Theorem C, $E_{\mathrm{p}}$ is $R_{p}$-free, and so from Proposition 2.3 we conclude that $\alpha\left(E_{\mathfrak{p}}\right)=0$. But $\alpha\left(E_{\mathfrak{p}}\right)=\alpha(E) \cdot R_{\mathfrak{p}}=0$, and since $R$ is a domain, $\alpha(E)=0$. Hence $E$ is $R$-free.

4. The ideal $\beta(E)$. Let $R$ be an arbitrary commutative ring and $E$ an arbitrary $R$-module. Define $\beta(E)$ to be the intersection of all the annihilators of the modules $\operatorname{Ext}_{R}^{1}(E, C)$ as $C$ runs through all $B$-modules. Clearly $\beta(E)=R$ if and only if $E$ is $R$-projective. It is well known that $x$ is in $\beta(E)$ if and only if given any epimorphism $f: P \rightarrow E$ there is a homomorphism $g: E \rightarrow P$ such that $f g$ is multiplication by $x$. For the sake of completeness we sketch a proof of this fact.

Clearly if the map $E \rightarrow^{x} E$ (multiplication by $x$ ) can be factored through a projective module, then $x \operatorname{Ext}_{R}^{1}(E, C)=0$ for any module $C$. On the other hand suppose $x \operatorname{Ext}^{1}(E, C)=0$ for any $R$-module $C$. Let $0 \rightarrow C \rightarrow P \rightarrow E \rightarrow 0$ be an exact sequence of $R$-modules. Then we get a commutative diagram, 


$$
\begin{aligned}
& \operatorname{Hom}(E, P) \rightarrow \operatorname{Hom}(E, E) \rightarrow \operatorname{Ext}^{1}(E, C) \\
& \downarrow x \quad \downarrow x \quad \downarrow x \\
& \operatorname{Hom}(E, P) \rightarrow \operatorname{Hom}(E, E) \rightarrow \operatorname{Ext}^{1}(E, C)
\end{aligned}
$$

with exact rows. Now the endomorphism of $E$ which is multiplication by $x$ is the image of the identity map in $\operatorname{Hom}(E, E)$ under the multiplication by $x$. Since $x \cdot \operatorname{Ext}^{1}(E, C)=0$, we have by usual diagram chasing that multiplication by $x$ in $\operatorname{Hom}(E, E)$ comes from $\operatorname{Hom}(E, P)$, which shows that the map $E \rightarrow{ }^{x} E$ can be factored through $P$ as desired. It is this latter description of $\beta(E)$ that we shall use throughout the rest of this note.

Proposition 4.1. Let $R$ be a domain and $E$ a finitely generated $R$-module. If $\alpha_{p}(E) \neq 0$, then $x^{p}$ is in $\alpha_{p}(E)$ for each $x$ in $\beta(E)$. Therefore $\beta(E)$ is contained in the radical of $\alpha_{p}(E)$ if $\alpha_{p}(E) \neq 0$.

Proof. Let $f: F \rightarrow E$ be an epimorphism with $F$ a finitely generated free $R$-module. Since $x$ is in $\beta(E)$ we know that there is a homomorphism $g: E \rightarrow F$ such that $f g: E \rightarrow E$ is multiplication by $x$. Therefore we have induced $\Lambda^{p} g: \Lambda^{p} E \rightarrow \Lambda^{p} F$ and $\Lambda^{p} f: \Lambda^{p} F \rightarrow \Lambda^{p} E$ such that $\Lambda^{p} f\left(\Lambda^{p} g\right)=\Lambda^{p}(f g): \Lambda^{p} E$ $\rightarrow \Lambda^{p} E$. Since $f g$ is multiplication by $x$ it follows that $\Lambda^{p}(f g)$ is multiplication by $x^{p}$. But by hypothesis $\alpha_{p}(E) \neq 0$ which means that $\Lambda^{p} E$ has a nontrivial annihilator. Since $\Lambda^{p} F$ is a free $R$-module we have that $\Lambda^{p} g: \Lambda^{p} E \rightarrow \Lambda^{p} F$ is the zero map, from which it follows that multiplication by $x^{p}$ applied to $\bigwedge^{p} E$ is the zero map, i.e. $x^{p}$ is in $\alpha_{p}(E)$.

Let $f: P \rightarrow E$ be an epimorphism where $P$ is $R$-projective. Then we have a map $f^{*}: \operatorname{Hom}_{R}(E, P) \rightarrow \operatorname{Hom}_{R}(E, E)$ and we have a ring homomorphism $t: R \rightarrow \operatorname{Hom}_{R}(E, E)$ where $t(x)=$ multiplication by $x$. We claim that $x$ is in $\beta(E)$ if and only if $t(x)$ is in $\operatorname{Im} f^{*}$. Now $t(x)$ is in $\operatorname{Im} f^{*}$ if and only if $t(x)$ can be factored through $P$, i.e. if and only if there is a $g: E \rightarrow P$ such that $f g=t(x)$. It is clear that if $t(x)$ can be factored through $P$, then $x$ is in $\beta(E)$. On the other hand if $x$ is in $\beta(E)$ it can be factored through $P$ since it can be factored through any module mapped onto $E$.

Now if we assume that $R$ is noetherian, $E$ is finitely generated, we can choose a finitely generated projective module $P$ which maps onto $E$. Since $E$ is finitely generated and $R$ is noetherian, we have that $R_{\mathfrak{p}} \otimes_{R} \operatorname{Hom}_{R}(E, C)$ $\approx \operatorname{Hom}_{R_{\mathfrak{p}}}\left(E_{\mathfrak{p}}, C_{\mathfrak{p}}\right)$ for all prime ideals $\mathfrak{p}$ in $R$. From this fact and the description of $\beta(E)$ given above, it follows that $\beta(E)_{\mathfrak{p}}=\beta\left(E_{\mathfrak{p}}\right)$. Since a module $E$ is projective if and only if $\beta(E)=R$ we have,

Proposition 4.2. If $R$ is a noetherian ring and $E$ is a finitely generated $R$ module, then a prime ideal $\mathfrak{p}$ contains $\beta(E)$ if and only if $E_{\mathfrak{p}}$ is not $R_{\mathfrak{p}}$-free.

We can now give an alternate proof of Theorem C. Let $R$ be a noetherian, local domain and $E$ a finitely generated $R$-module. If $\mathfrak{p}$ is a prime ideal such 
that $E / \mathfrak{p} E$ is $R / \mathfrak{p}$-free, then $\mathfrak{p}$ contains $\alpha(E)$. Now if $\alpha(E) \neq 0$, then $\beta(E)$ is contained in the radical of $\alpha(E)$ and therefore $p$ contains $\beta(E)$. But that means that $E_{\mathfrak{p}}$ is not $R_{\mathfrak{p}}$-free (see Proposition 4.2). Therefore if $E / \mathfrak{p} E$ is $R / \mathfrak{p}$-free and $E_{\mathfrak{p}}$ is $R_{\mathfrak{p}}$-free, then $\alpha(E)=0$ and therefore by Proposition $2.3 E$ is $R$-free, which establishes Theorem $\mathrm{C}$.

\section{REFERENCES}

1. M. Auslander and D. Buchsbaum, On ramification theory in noetherian rings, Amer. J. Math. 81 (1959), 749-765.

2. H. Cartan and S. Eilenberg, Homological algebra, Princeton Univ. Press, Princeton, N. J., 1956.

3. C. Chevalley, Fundamental concepts of algebra, Academic Press, New York, 1956.

BRANDEIS UNIVERSITY,

Waltham, Massachusetts 